\section{AB0141 THE ROLE OF AIR POLLUTION ON EXTRACELLULAR VESICLES AS A POTENTIAL PRO-INFLAMMATORY STIMULUS IN RHEUMATOID ARTHRITIS}

Tommaso Schioppo ${ }^{1}$, Mirjam Hoxha ${ }^{2}$, Simona lodice ${ }^{2}$, Laura Pergoli ${ }^{2}$, Orazio De Lucia $^{1}$, Antonella Murgo ${ }^{1}$, Luca Ferrari ${ }^{2}$, Valentina Bollati ${ }^{2}$,

Francesca Ingegnoli ${ }^{1,2}$. ${ }^{1}$ ASST Pini-CTO, Clinical Rheumatology, Milano, Italy, ${ }^{2}$ Università degli Studi di Milano, Department of Clinical Sciences and Community Health, Milano, Italy

Background: Rheumatoid arthritis (RA) is an heterogeneous chronic autoimmune disorder potentially leading to a progressive joint damage with great impact on quality of life. RA pathogenesis is complex and involves environmental factors that trigger disease in genetically susceptible individuals. Extracellular vesicles (EVs) have been described to play an important role in RA pathogenesis and to modulate autoimmune response following environmental exposures, such as air pollution.

Objectives: Our aim was to evaluate the effects of particulate matter (PM) with aerodynamic diameter $\leq 10 \mu \mathrm{m}\left(\mathrm{PM}_{10}\right)$ and $\leq 2.5 \mu \mathrm{m}\left(\mathrm{PM}_{2.5}\right)$ on $\mathrm{EVs}$ in $\mathrm{RA}$ and osteoarthritis $(\mathrm{OA})$ as control.

Methods: Plasma EVs were analyzed by Nanosight and flow cytometry: CD14 (monocyte/macrophage), CD61 (platelet), CD25 (T-reg), human endogenous retrovirus w (HERV-w), human leukocyte antigen G (HLA-G). Demographic and clinical data were collected for each patient. Plasma EV concentrations were measured in RA and OA patients and were analyzed by generalized linear regression models. Daily PM concentrations, estimated by Regional Environmental Protection Agency at municipality resolution, were used to assign short-term exposure (mean of the 7 days preceding the evaluation) to each patient.

Results: 12 consecutive patients with RA (median age 68.1, median disease duration 9.3, 12 female, median DAS28 2.25, 5 positive for rheumatoid factors, 6 positive for anti-citrullinated peptide antibodies) and 12 patients with OA (median age 67.1, median disease duration 9.3, 8 female) were enrolled. Analysis of EVs concentration, according to their dimensions, showed a negative association of exosomes $(63-92 n m)$ in RA compared to $O A(p<0.05)$. The increase of $P_{2.5}$ led to a decrease of $C D 14+$ microvesicles $(M V)(\beta=-0.13 ; p<0.01)$ and $C D 61+(\beta=-$ $0.08 ; p=0.05)$ in $R A$, and of HERV-w in OA ( $\beta=-0.09 ; p=0.01)$. Similar results were observed analyzing PM10 exposure. PM exposure was not observed to modify $\mathrm{CD} 25+$ and HLA-G+ MV release both in RA and OA patients (table below). Moreover, we compared plasmatic EVs mean concentration among patients with RA and $O A$, and we found a significant difference in the two groups in the HERV-w subpopulations ( $\beta_{A R}$ vs $\beta_{O A}=0.044$ vs $-0.091 ; p=0.011$ ). In RA patients we also observed a significant association between EVs (CD14+ and HLA-G+ MV) and DAS28 ( $\beta_{\mathrm{CD} 14+}=0.03 ; p=0.01$ and $\beta_{\text {HLA }-G+}=0.04 ; p=0.02$ ). Finally, we observed a negative association between exosomes and $\mathrm{C}$-reactive protein (CRP) $(\beta=-1.99 ; p=0.03)$, and a positive association between HERV-w and Erythrocyte Sedimentation Rate (ESR) $(\beta=0.53 ; p=0.06)$, and $\mathrm{HLA}-\mathrm{G}+$ and $\mathrm{ESR}$ $(\beta=0.29 ; p=0.01)$.

\begin{tabular}{|c|c|c|c|c|c|c|}
\hline \multirow[t]{2}{*}{ EV } & \multicolumn{3}{|c|}{ RA } & \multicolumn{3}{|c|}{ OA } \\
\hline & $\bar{\beta}$ & SE & $\begin{array}{c}\text { P- } \\
\text { value }\end{array}$ & $\beta$ & SE & $\begin{array}{c}\text { P- } \\
\text { value }\end{array}$ \\
\hline \multicolumn{7}{|c|}{$\begin{array}{l}\mathrm{PM}_{2.5} \text { exposure } \\
\text { EV count }\end{array}$} \\
\hline Total EV & -0.04 & 0.02 & 0.08 & 0.02 & 0.03 & 0.49 \\
\hline Exosomes & -0.01 & 0.02 & 0.60 & 0.01 & 0.03 & 0.66 \\
\hline MV & -0.02 & 0.02 & 0.36 & 0.01 & 0.03 & 0.66 \\
\hline \multicolumn{7}{|c|}{ MV subtypes } \\
\hline CD14+ & -0.13 & 0.03 & $<0.01$ & -0.04 & 0.04 & 0.25 \\
\hline CD61+ & -0.08 & 0.04 & 0.05 & 0.00 & 0.05 & 0.99 \\
\hline CD25+ & -0.02 & 0.02 & 0.48 & -0.02 & 0.03 & 0.48 \\
\hline HERV-w+ & 0.04 & 0.03 & 0.08 & -0.09 & 0.04 & 0.01 \\
\hline HLA-G+ & -0.03 & 0.02 & 0.18 & -0.01 & 0.03 & 0.67 \\
\hline \multirow{2}{*}{\multicolumn{7}{|c|}{$\mathrm{PM}_{10}$ exposure }} \\
\hline EV count & & & & & & \\
\hline Total EV & -0.01 & 0.01 & 0.52 & 0.02 & 0.02 & 0.43 \\
\hline Exosomes & -0.02 & 0.02 & 0.26 & 0.04 & 0.03 & 0.14 \\
\hline MV & -0.01 & 0.01 & 0.63 & 0.02 & 0.03 & 0.52 \\
\hline \multicolumn{7}{|c|}{ MV subtypes } \\
\hline CD14+ & -0.09 & 0.02 & $<0.01$ & -0.01 & 0.04 & 0.72 \\
\hline CD61+ & -0.06 & 0.03 & 0.02 & -0.02 & 0.05 & 0.76 \\
\hline CD25+ & -0.01 & 0.01 & 0.53 & -0.02 & 0.03 & 0.50 \\
\hline HERV-w+ & 0.03 & 0.02 & 0.07 & -0.07 & 0.04 & 0.05 \\
\hline $\mathrm{HLA}-\mathrm{G}_{+}$ & -0.01 & 0.02 & 0.46 & 0.00 & 0.03 & 0.97 \\
\hline
\end{tabular}

Conclusion: The results of this pilot study show that PM exposure modulates the release of EVs carrying HLA-G and/or HERV-w in RA patients. This might be interpreted as an attempt of immune system to counteract the perturbation provoked by a pro-inflammatory environmental stimulus. More research is still needed to tie the genetic, epigenetic and environmental factors together and to determine their roles in RA pathogenesis.

Disclosure of Interests: None declared

DOI: 10.1136/annrheumdis-2019-eular.2180

\section{$\mathrm{AB} 0142$ \\ IMMUNOREGULATORY ROLES OF $\mathrm{B}^{2}$ INTEGRINS IN RHEUMATOID ARTHRITIS}

Leonie Schittenhelm $^{1,2,3}$, Jamie Robertson ${ }^{1,3}$, Arthur Pratt ${ }^{2,3}$, Catharien Hilkens ${ }^{2,3}$, Vicky L. Morrison ${ }^{1,3}$. ${ }^{1}$ Institute of Infection, Immunity and Inflammation, Glasgow, United Kingdom; ${ }^{2}$ Institute of Cellular Medicine, Newcastle, United Kingdom; ${ }^{3}$ Arthritis Research UK Rheumatoid Arthritis Centre for Excellence (RACE), Glasgow, United Kingdom

Background: Evidence is mounting that $\beta^{2}$ integrins, adhesion receptors that exist in a continuum between 'inactive' and 'active', facilitate not only inflammation but are also important in immune regulation. Elucidating their role on dendritic cells (DCs) is hereby of special interest due to the pivotal role DCs play in immune activation and tolerance. Previous research suggests that $\beta^{2}$ integrin-mediated immunoregulation is prominent in murine $\mathrm{DCs}^{1}$, but a similar role in human cells has yet to be characterised.

Objectives: We hypothesised that altered $\beta^{2}$ integrin expression, activation or function in DCs may contribute to the immune dysregulation prevalent in autoimmune disorders such as Rheumatoid Arthritis (RA). To this end, we aimed to characterise $\beta^{2}$ integrin expression and activation in antigen-presenting cell (APC) populations from people with RA compared to healthy controls.

Methods: Using flow cytometry, we can detect both total and activated $\beta^{2}$ integrin subunits (CD11a, CD11b, CD18) on different APC populations present in human blood and synovial fluid.

Results: Based on our results so far, we found CD11b expression to be equivalent in APC populations between patients with RA and healthy controls. However, expression of both the total and active forms of CD11a was reduced across several APC populations in RA patients compared to healthy controls, with cDC2s $(\mathrm{CD} 1 \mathrm{c}+)$ showing the most striking reduction. Interestingly, we found that $\mathrm{CDC} 2 \mathrm{~s}$ from matched RA synovial fluid samples express even lower levels of CD11a than blood cDC2s. Previous studies show that $\mathrm{CDC} 2 \mathrm{~s}$ are the DC population predominant in the RA joint, characterised by an increased capacity to attract $T$ cells to the site of inflammation and activate self-reactive T cells ${ }^{2}$. As CD11a is described to have largely pro-inflammatory effects by contributing to immune cell contacts and recruitment to inflammatory sites, this could suggest that contrary to our initial hypothesis, cells downregulate CD11a as a means of limiting the aberrant inflammation present in the disease.

Furthermore, cDC2s from RA patients in remission express lower levels of active CD11a compared to those from patients with high disease activity. Upon stimulation of cDC2s with PMA, cells from patients with active disease decrease their activation of CD11a, while neither healthy controls nor patients in remission show any change in CD11a activation. This might suggest that CD11a activation is differentially regulated in patients with active disease, while remission signifies a return to a state of normal CD11a regulation on cDC2s. In additional work on exploring monocyte-derived DCs (MO-DCs) as a potential therapeutic for RA, we did not detect a reduction in CD11a expression in activated MO-DCs, suggesting that the effect may be specific to settings of chronic inflammation that cannot be easily modelled in vitro.

Conclusion: Further interrogation of our integrin expression data, including dis ease stratification, together with functional studies on RA patient cDC2s compared to MO-DCs, is currently under way to fully investigate the role of this immunoregulatory pathway in autoimmune disorders. In doing so, we will explore $\beta^{2}$ integrin subunits and signalling pathways as potential therapeutic targets in RA.

\section{REFERENCES}

[1] Morrison, Vicky Louise, et al. "Loss of beta2-integrin-mediated cytoskeletal linkage reprogrammes dendritic cells to a mature migratory phenotype." Nature communications 5 (2014): 5359.

[2] Moret, Frederique M., et al. "Intra-articular CD1c-expressing myeloid dendritic cells from rheumatoid arthritis patients express a unique set of $T$ cellattracting chemokines and spontaneously induce Th1, Th17 and Th2 cell activity." Arthritis research \& therapy 15.5 (2013): R155.

Disclosure of Interests: Leonie Schittenhelm: None declared, Jamie Robertson: None declared, Arthur Pratt Grant/research support from: Dr. Pratt is in receipt of an externally peer-reviewed Investigator Initiated Research grant from Pfizer (£66,000)., Grant/research support from: Dr. Pratt is in receipt of an externally peer-reviewed Investigator Initiated Research grant from Pfizer $(£ 66,000)$. Speakers bureau: Dr Pratt has received honoraria from Eli Lilly and Janssen-Cilag Ltd. for his time in preparing presentations for non-promotional meetings that have been paid directly to Newcastle University., Speakers bureau: Dr Pratt has received honoraria from Eli Lilly and Janssen-Cilag Ltd. for his time in preparing presentations for non-promotional meetings that have been paid directly to Newcastle University., Catharien Hilkens: None declared, Vicky L. Morrison: None declared

DOI: 10.1136/annrheumdis-2019-eular.5465 